\title{
Projet autoroutier A 89
}

La-Tour-de-Salvagny-Balbigny (Rhône-Loire)

Sylvain Motte, Emmanuel Ferber, Véronique Monnoyeur-Roussel et Catherine Bellon

\section{(2) OpenEdition}

Édition électronique

URL : http://journals.openedition.org/adlfi/1681

ISSN : 2114-0502

Éditeur

Ministère de la culture

Référence électronique

Sylvain Motte, Emmanuel Ferber, Véronique Monnoyeur-Roussel et Catherine Bellon, « Projet

autoroutier A 89 », ADLFI. Archéologie de la France - Informations [En ligne], Rhône-Alpes, mis en ligne le 01 mars 2008, consulté le 01 mai 2019. URL : http://journals.openedition.org/adlfi/1681

Ce document a été généré automatiquement le 1 mai 2019.

(C) Ministère de la Culture et de la Communication, CNRS 


\title{
Projet autoroutier A 89
}

\author{
La-Tour-de-Salvagny-Balbigny (Rhône-Loire) \\ Sylvain Motte, Emmanuel Ferber, Véronique Monnoyeur-Roussel et \\ Catherine Bellon
}

Identifiant de l'opération archéologique : 9704

Date de l'opération : 2008 (EV)

1 Le tracé de l'A 89 long de $48 \mathrm{~km}$ traverse deux départements et représente une emprise totale d'environ 600 ha. L'ensemble des opérations archéologiques, diagnostics et fouilles préventives, doit s'inscrire entre 2007 et 2010.

En concertation avec l'aménageur et le calendrier des travaux prévus, le tracé du projet autoroutier a été divisé en 12 tronçons. Chacun est autonome et fait l'objet d'un rapport de diagnostic distinct.

En 2008, 6 diagnostics ont été réalisés :

4 Dans le Rhône : sur les secteurs 2, 3 et 5 - secteur 2 (Fleurieu-sur-l'Arbresle) - secteur 3 (de Châtillon-d'Azergues à Saint-Germain-sur-l'Arbresle) - secteur 5 (de Pontcharra-surTurdine à Saint-Romain-de- Popey)

Dans la Loire : sur les secteurs 10,11,12 - secteur 10 (Néronde à Sainte-Colombe-surGand) - secteur 11 (de Balbigny à Néronde) - secteur 12 (échangeur de Balbigny ).

Par ailleurs une fouille a été réalisée par l'Inrap côté Rhône sur le secteur 8 au lieu-dit Boussuivre, commune de Joux.

GAIDON-BUNUEL Marie-Agnès, LENOBLE Michel et ROYET Robert Ministère de la Culture

Secteur 2 : FLEURIEUX-SUR-L'ARBRESLE

9 Le secteur 2 du projet autoroutier traverse la commune de Fleurieux-sur-L'ArbresIe sur 3,6 km. Cinq cents sondages mécaniques ont permis de vérifier la présence d'éventuels sites archéologiques. Le tracé étudié traverse un paysage rural où dominent prés et bois entrecoupés par quelques terres agricoles. La topographie est marquée par une légère pente vers le nord et la vallée de la Brévenne. La stratigraphie d'ensemble est 
simple: le substrat rocheux primaire est recouvert, la plupart du temps, par une formation alluviale ancienne et sur ces cailloutis reposent des colluvions épaisses de 0,30 $\mathrm{m}$ à $0,50 \mathrm{~m}$, puis la terre végétale.

10 Trois sites A, B et C ont été mis au jour sur le secteur 2. Sur le site A, la présence d'une couche à tegulae, de fragments de roche et de quelques tessons de céramique témoigne d'une occupation antique clairement délimitée sur environ $4500 \mathrm{~m}^{2}$. Le site a presque totalement été détruit par les pratiques agraires dont les traces sont bien visibles. Seuls sont conservés un puits, un solin de mur en galets, deux fosses et un fossé. Ce puits n'était pas comblé, protégé par un couvercle de pierres. Le cuvelage de blocs, sans liant, est profond de $17 \mathrm{~m}$. Le rare mobilier céramique permet d'évaluer une occupation comprise entre le $\mathrm{I}^{\text {er }}$ siècle et le Ille siècle de notre ère.

11 Sur le site B, l'occupation antique est bien circonscrite par un mur d'enceinte connu sur trois côtés délimitant un rectangle de $1700 \mathrm{~m}^{2}$. Le mobilier de surface montre que le site se développe sur quelques décamètres hors emprise vers le nord. L'occupation est caractérisée par au moins onze murs de blocs de roche verte basaltique. Le plan général défini un espace clos dont la partie orientale présente des divisions internes, la partie occidentale étant un espace ouvert ou dévolu à des aménagements modestes. Au moins un mur présente une élévation dont deux assises sont conservées; il délimite le sol d'un espace certainement surbaissé. D'après le rare mobilier issu des sondages et de la prospection pédestre, ce petit établissement agricole a été occupé entre le $\mathrm{I}^{\mathrm{er}}$ siècle et le Ill ${ }^{e}$ siècle de notre ère.

12 Le site $\mathrm{C}$ d'environ $230 \mathrm{~m}^{2}$ a été mis au jour à $250 \mathrm{~m}$ à l'ouest du site B. Fouillé exhaustivement, lors du diagnostic, il comprend deux petites constructions mitoyennes à couverture de tuiles, constituées de trois murs de plan en U. Ces deux bâtiments sont construits à proximité d'un puits circulaire profond de plus de $3,5 \mathrm{~m}$. Des fossés de drainage délimitent l'ensemble au nord et au sud. La destination de ces deux édicules n'est pas connue, mais la proximité du site $\mathrm{B}$, considéré comme une petite exploitation agricole, et la présence du puits, permettent d'interpréter ces vestiges comme une dépendance agricole ou artisanale. Les quelques tessons recueillis témoignent d'une période d'occupation synchrone de celle du site $B$ ( ${ }^{\text {er }}$ siècle au Ille siècle de l'ère).

\section{Secteur 3 phase 1 : CHATILLON-D'AZERGUES Coleymieux}

14 La colline de Coleymieux doit servir à l'implantation d'une tête de pont du viaduc franchissant la Brévenne. A cet endroit, l'emprise du tracé s'élargit $\left(635010 \mathrm{~m}^{2}\right)$ afin de pouvoir accueillir, outre l'accès à l'ouvrage d'art, un dépôt et un bassin de décantation.

15 Cette colline est connue pour receler plusieurs occupations préhistoriques: un racloir daté du moustérien et une cinquantaine de silex appartenant à la période néolithique y ont été mis au jour à la fin du XIX ${ }^{\mathrm{e}} \mathrm{s}$. Des crémations antiques localisées et une chapelle dédiée à Saint-Martin mentionnée en 1262 sont aussi recensées sur ce secteur.

16 À l'issue du diagnostic, aucun site en place n'a été mis mis au jour. En revanche, des sondages pratiqués dans un talweg ont permis de découvrir un tressage de paléochenaux dont les comblements contenaient du mobilier médiéval (essentiellement céramique, 
mais un objet en fer et un fragment de meule en basalte ont aussi été recensés). Un site datable des $\mathrm{X}^{\mathrm{e}} \mathrm{s}$.au XII ${ }^{\mathrm{e}} \mathrm{s}$. et au moins en partie démantelé lors de diverses crues pourrait être situé en amont de la zone sondée sans qu'il ne soit possible d'en préciser l'origine exacte et sa nature.

\section{Secteur 3 phase 2 : CHATILLON-D'AZERGUES et SAINT-GERMAIN-SUR-L'ARBRESLE}

Ce secteur cumule $306449 \mathrm{~m}^{2}$. La phase 2, ici présentée, concerne $242939 \mathrm{~m}^{2}$. Sur cette surface, des parcelles n'ont pu être sondées, soit parce qu'elles étaient inondées, soit faute d'accord des propriétaires. neuf mille cent dix-neuf mètres carrés ont été sondés sur les $160770 \mathrm{~m}^{2}$ accessibles soit 5,6\% de la surface. Cent quarante-cinq sondages ont été réalisés sur Saint-Germain-sur-l'Arbresle et cent soixante-cinq sur la commune de Châtillon-d'Azergues. Les sondages ont été menés jusqu'au terrain naturel. Ils sont peu profonds car le recouvrement stratigraphique est faible. Deux aménagements ont été mis au jour :

- une structure en creux correspondant soit à une fosse d'extraction soit à un chablis sur la commune de Châtillon-d'Azergues ;

- un aménagement de type mare ou fosse d'extraction sur la commune de Saint-Germainsur-l'Arbresle.

21 Quelques silex épars ont été également trouvés sur la commune de Châtillon-d'Azergues. Ces découvertes correspondent très vraisemblablement à des objets isolés perdus lors de déplacement.

22 Ce quasi vide archéologique peut s'expliquer par l'éloignement du secteur des axes de circulation que forment les vallées de la Brévenne et de l'Azergues. On peut imaginer bien sûr qu'une occupation sommaire a pu disparaître en raison du faible recouvrement stratigraphique et $\mathrm{du}$ brassage des terrains par l'agriculture ou par l'altération géologique. Cependant, aucun autre mobilier, toutes périodes confondues et même en position secondaire, n'a été repéré sur l'ensemble du secteur.

\section{Secteur 5 : PONTCHARRA-sur-TURDINE et SAINT- ROMAIN-de-POPEY}

Le secteur 5 débute sur la commune de Pontcharra-sur-Turdine et se poursuit sur celle de Saint-Romain-de-Popey. Si jusqu'à maintenant aucun site n'a été recensé sur l'emprise même de l'autoroute, les deux communes ont néanmoins livré quelques indices d'occupation pour différentes périodes. Le site de Miolan à Pontcharra-sur-Turdine et le site du Crêt de Popey à Saint-Romain-de-Popey renferment tous deux des vestiges de la Préhistoire et de l'époque antique. Rappelons que la voie romaine de Lyon à Roanne pourrait reprendre le tracé de la RN 7, avec une étape à Mediolanum,identifié à Miolan. La paroisse de Saint-Romain-de-Popey, dépendante de l'abbaye de Savigny, est quant à elle mentionnée dès le Moyen Âge. 

communales. du parement en élévation. peu, marécageux. le grand chenal appartiendrait au XVIII ${ }^{\mathrm{e}} \mathrm{s}$. été abandonnés. surcreusement des sols internes. solides?
Cent vingt-cinq sondages ont été effectués sur la commune de Pontcharra-sur-Turdine et trois cent quarante-huit sur celle de Saint-Romain-de-Popey. Deux sites ont ainsi été mis en évidence Saint-Romain-de-Popey : un bâtiment octogonal et un établissement galloromain. Des vestiges plus épars ou ténus permettent d'appréhender l'occupation du territoire pour les périodes les plus anciennes et de renseigner les cartes archéologiques

Dans la parcelle 973, sondage 669, un bâtiment de plan octogonal a été mis au jour. Il est conservé sur une ou deux assises en fondation et sur deux côtés subsistent des portions

Ce bâtiment s'élève sur le lit majeur de la Turdine en rive gauche de la rivière qui est décalée contre le versant de la rive droite. Les terrains inondables étaient, il y a encore

L'étude a montré la présence d'anciens chenaux dont le plus grand se trouve à l'emplacement de deux segments détruits de la construction. Très peu d'éléments subsistent pour identifier et dater ce site. Quelques tessons de céramiques communes grises et communes rouges ramassés lors du nettoyage des murs peuvent être attribués au bas Moyen Âge. Néanmoins l'aspect fragmentaire de ces éléments et l'absence de fragments typologiques ne permettent de dater que de façon très large entre le XlV ${ }^{\mathrm{e}} \mathrm{s}$. et le $\mathrm{XVI}^{\mathrm{e}} \mathrm{s}$. La céramique découverte dans les derniers comblements qui viennent fossiliser

L'état d'arasement du site est tel que l'hypothèse d'une construction inachevée est envisagée. Une grande crue aurait touché le site en début des travaux qui auraient alors

La fonction de ce bâtiment est hypothétique ? Plusieurs suppositions sont possibles parmi lesquelles on peut citer un pigeonnier, un moulin, une tour ou un donjon pouvant dépendre du château d'Avauges qui s'élève à peu de distance de là.

Le second site concerne un grand bâtiment rectangulaire de $12 \mathrm{~m}$ sur $10 \mathrm{~m}$. Montés en blocs de granite sans liant, les murs sont conservés en fondation. Le bâtiment possède au moins trois espaces. Des massifs en façade occidentale laissent supposer la présence d'un porche. L'observation des maçonneries montre deux états de construction. Les vestiges conservés évoquent une construction de $12 \mathrm{~m}$ x $10 \mathrm{~m}$ aux murs étroits $(0,60 \mathrm{~m})$. Des trous de poteau, repérés à l'intérieur des blocages des murs, laissent supposer une construction mixte, bois et pierre, peut-être associée à de la terre ou du torchis.

Un doublage intérieur des murs ouest caractérise un second état. Ce doublage est réalisé avec de gros blocs de granite équarris sur leur face externe. Il semble contemporain d'un

Le doublage a eu très certainement des conséquences architecturales sur l'élévation du bâti. Est-on passé d'une architecture en matériaux légers à une architecture plus exclusivement en pierre? L'élévation occidentale était-elle plus haute que le reste du bâti ? La couverture à cet endroit plus lourde, d'où la nécessité de murs porteurs plus

34 Outre ce bâtiment, le sondage a mis en évidence l'existence de niveaux de circulation ainsi que des fosses, des fossés, un caniveau et surtout une concentration de trous de poteau au sud du bâti. Ces éléments n'ont pas été fouillés mais on a pu observer que la plupart d'entre eux possède des éléments de calage. Certains alignements de poteaux se 
dessinent, mais ils ne peuvent être tenus pour certains tant que la surface n'a pas été intégralement fouillée, le niveau de sol dans lequel ils sont installés et les niveaux de démolition qui recouvrent une partie du bâti mitoyen peuvent encore occulter d'autres aménagements de ce type.

Dans l'état actuel de la recherche, la chronologie entre le bâti maçonné et les structures légères ne peut être définie avec certitude. En confrontant, l'étude archéologique et l'étude de la céramique, on peut proposer l'hypothèse d'un établissement précoce sur poteaux, peut-être dès le $\mathrm{I}^{\mathrm{er}} \mathrm{s}$. av. J.-C., auquel succède au $\mathrm{I}^{\mathrm{er}}$ siècle de notre ère, éventuellement après une phase d'abandon, un bâtiment fondé. La fonction de ces différentes occupations n'a pas été encore tranchée.

La partie située à l'est de cette parcelle a mis en évidence la présence de nombreux drains ou fossés, toutes périodes confondues. Certains sont modernes, mais d'autres sont antiques. De même, des alignements de murs, très arasés, ont également été observés dans ce même secteur. Dans un des sondages, une concentration de matériel céramique, dont la datation s'échelonne du $\mathrm{I}^{\mathrm{er}}$ s. av. J.-C. au III $\mathrm{e}$ s. apr. J.-C., a été localisée dans une couche hydromorphe. Cette accumulation pourrait signaler l'emplacement d'un dépotoir ou d'un épandage à proximité d'une occupation, voire d'un habitat. L'importance du matériel tendrait à prouver la densité d'une occupation antique dans le secteur du Pont d'Avauges.

Il est également à noter que toute cette zone présente une faible épaisseur stratigraphique témoin d'une érosion importante qui pourrait être le signe d'une forte occupation à l'âge du Fer, dans une zone relativement horizontale et humide.

Tout au long du tracé, les sondages ont mis au jour des tessons de céramique médiévale et moderne. La variété des périodes chronologiques qu'illustrent ces poteries, ne plaide pas non plus en faveur d'une occupation définie à proximité du lieu de leur découverte.

Du matériel céramique néolithique et protohistorique dans des espaces bien délimités pourrait, par contre, témoigner de zones d'occupation très anciennes dans ou à proximité du tracé. Bien qu'identifiées sur la seule base de discriminations typochronologiques du mobilier céramique, fautes de structurations et de contextes sédimentaires anthropiques identifiés, différentes périodes ont pu être reconnues. Il semble bien que l'occupation du territoire entre les communes de Pontcharra-sur-Turdine et Saint-Romain-de-Popey soit pérennisée, du Néolithique moyen à la romanisation, étant bien sur entendu que, au travers de cette ébauche de lecture, des hiatus demeurent pour des pans entiers de la Protohistoire. Des occupations sur des flancs peu accentués sont pressenties soit pérénisées en amont des zones sondées. Les divagations de la Turdine et les phénomènes d'arasement ou de glissement de pentes n'ont donc pas complètement altéré les vestiges résiduels. La mise à jour de site en bon état de conservation dans cette zone n'est pas exclue pour ces périodes.

\section{Secteur 8 : JOUX Boussuivre}

Le projet autoroutier A 89 a conduit à intervenir sur une zone de $3000 \mathrm{~m}^{2}$ au lieu-dit Boussuivre après une campagne de diagnostics positifs en 2007. 
43 Au contact des monts du Lyonnais et du haut Beaujolais, la zone étudiée s'insère dans les monts de Tarare formant une barrière qui s'élève à plus de $900 \mathrm{~m}$ d'altitude entre les vallées de la Saône et du Rhône à l'est et la vallée de la Loire à l'ouest. Le site de Boussuivre se trouve dans la partie amont du vallon du même nom, affluent de La Turdine, et sur le bas de pente d'une autre combe affluente drainée par un ru non pérenne. Son versant atteint 14 ? (25\%) de pente nord-nord-ouest-sud-sud-est en amont et au droit du gisement. Situé en rive gauche et à $5 \mathrm{~m}$ du ru affluent, le site archéologique a subi partiellement son influence ainsi que celle de la forte pente. Ceci se traduit par des phénomènes d'érosion qui n'ont cependant pas totalement effacé une fréquentation de la fin de la Préhistoire (outils en silex du Néolithique), ainsi que des installations du second âge du Fer (II ${ }^{\mathrm{e}}$ s. et $\mathrm{I}^{\mathrm{er}}$ s. av. J.-C.) et du Moyen Âge (X ${ }^{\mathrm{e}}$ s. et $\mathrm{XI}^{\mathrm{e}} \mathrm{s}$.), qui se superposent. Les principales découvertes concernent les vestiges de plusieurs bâtiments datés de l'époque gauloise de La Tène finale (C2/D1 : $\mathrm{II}^{\mathrm{e}} \mathrm{s}$. et $\mathrm{I}^{\mathrm{er}} \mathrm{s}$. av. J.-C.) (Fig. $\mathrm{n}^{\circ} 1$ : Bâtis de la Tène finale) construits pour certains sur poteaux porteurs, pour d'autres sur sablières basses, au droit de petites terrasses aménagées dans cette forte pente. Parmi les artefacts mis au jour, outre quelques objets métalliques (clous, plaque, tenon, couteau) et de la céramique, on notera la présence d'amphores italiques.

Postérieurement à cette occupation gauloise, probablement de courte durée (1 ou 2 générations), une fréquentation gallo-romaine proche est attestée par la découverte, lors des sondages, de tuiles antiques, juste en amont du site, ainsi que par un unique fragment de verre sur la fouille.

46 Il faudra ensuite attendre le Moyen Âge ( $\mathrm{X}^{\mathrm{e}} \mathrm{s}$. et $\mathrm{XI}^{\mathrm{e}} \mathrm{s}$.) pour retrouver une nouvelle occupation marquée par deux installations successives. La première est caractérisée par une aire d'ensilage composée de quatorze fosses silos et trois structures à combustion, puis lui succèdent un bâtiment d'habitation associé à quelques aménagements annexes - enclos et structures en creux dont deux fonds de cabane. (Fig. $\mathrm{n}^{\circ} 2$ : Four XIe siècle)

47 Ces découvertes confirment l'essor démographique et la plus grande densité de l'occupation des campagnes que l'archéologie a permis de détecter à partir de l'an mil.

48 BELLON Catherine

49 INRAP

\section{ANNEXES}


Fig. $\mathrm{n}^{\circ} 1$ : Bâtis de la Tène finale

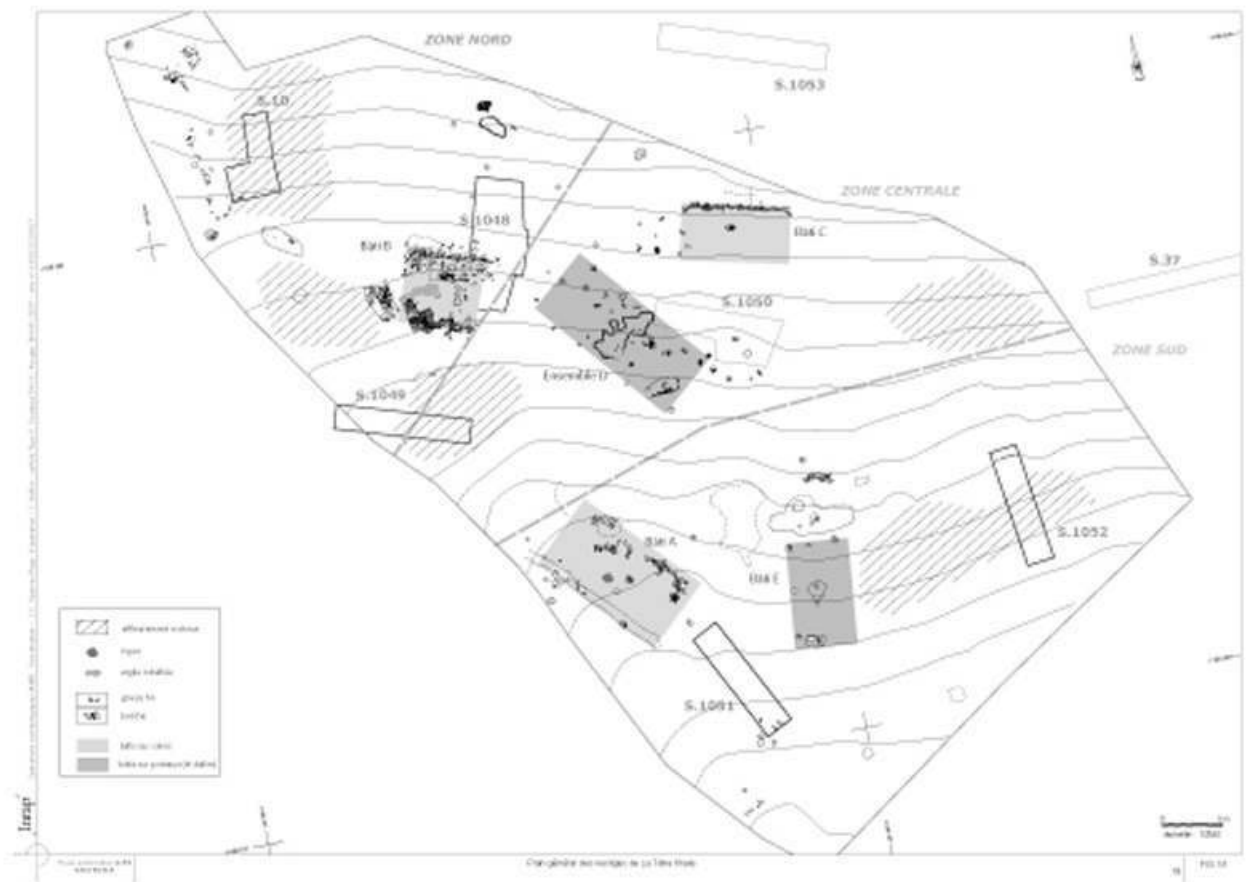

Auteur(s) : Bellon, Catherine (Inrap). Crédits : C. Bellon INRAP (2008)

Fig. $n^{\circ} 2$ : Four Xle siècle

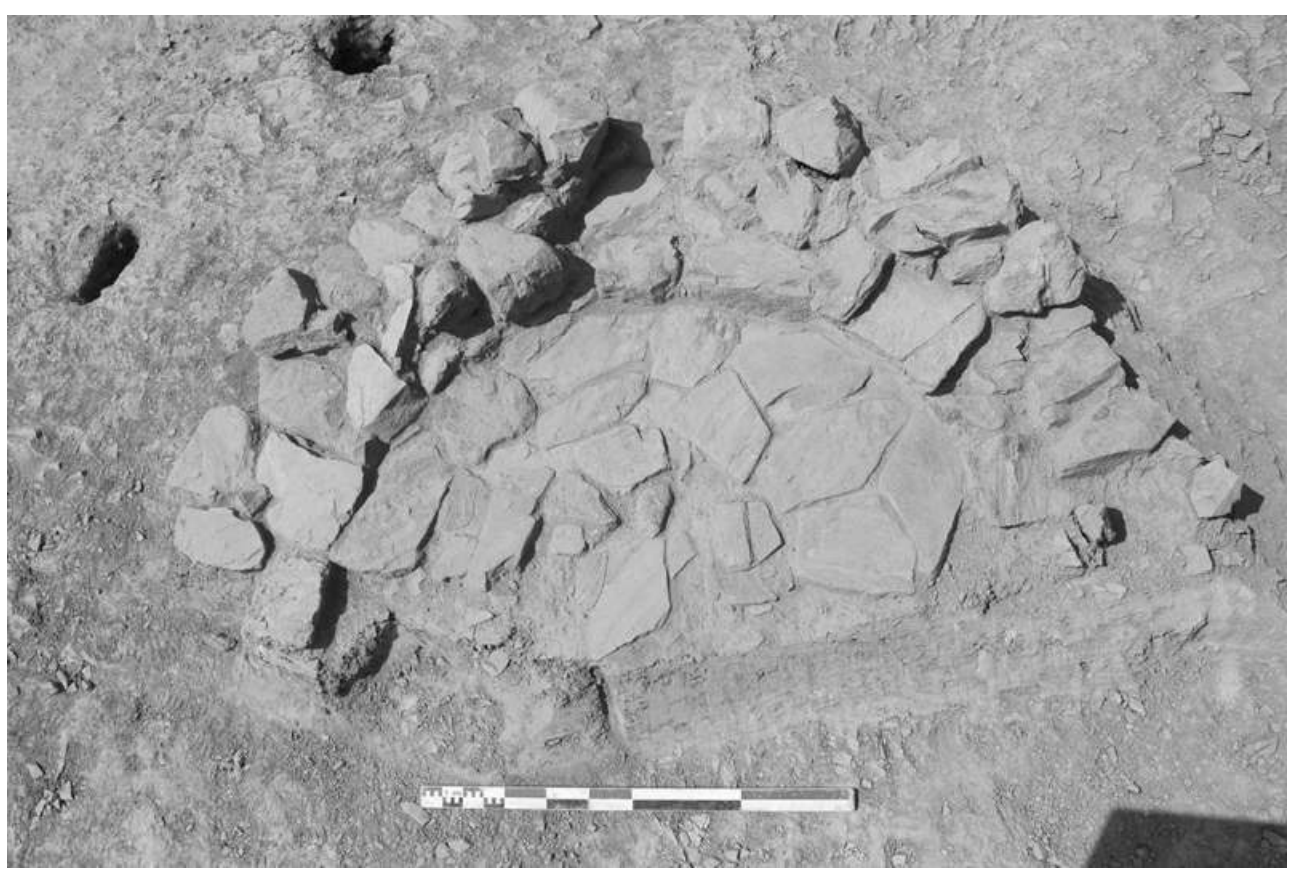

Auteur(s) : Bellon, Catherine (Inrap). Crédits : C. Bellon Inrap (2008) 
INDEX

operation Fouille d'évaluation (EV)

Index chronologique : âge du Fer, bas Moyen Âge, Empire romain, Ier siècle av. J.-C., Moyen Âge, Xe siècle apr. J.-C., XIe siècle apr. J.-C., XIIe siècle apr. J.-C., XIVe siècle apr. J.-C., XVIIIe siècle apr.

J.-C.

Index géographique : Rhône-Alpes, Rhône (69), Fleurieux-sur-l'Arbresle

Thèmes : amphore italique, céramique, céramique commune, céramique médiévale, fond de cabane, fosse, four, silex, trou de poteau

\section{AUTEURS}

\section{SYLVAIN MOTTE}

INRAP

EMMANUEL FERBER

Inrap

VÉRONIQUE MONNOYEUR-ROUSSEL

Inrap

\section{CATHERINE BELLON}

Inrap 